\section{Membrane-targeting AM-0016 kills mycobacterial persisters and shows low propensity for resistance development}

\author{
Devika Mukherjee', Hanxun Zou², Shouping Liu' ${ }^{2,3}$, Roger Beuerman²,3,4 \\ \& Thomas Dick ${ }^{* 1}$
}

\begin{abstract}
Aim: To test the hypothesis that targeting the cytoplasmic membrane may be an effective way to kill persister mycobacteria and delay the emergence of resistance. Methods: In vitro activity of AM-0016, a novel xanthone-based antibacterial, was assessed against growing and persister tubercle bacilli. Resistance mutation frequencies were determined. Biochemical membrane and electron microscopic analyses were carried out. Results: AM-0016 rapidly sterilized growing tubercle bacillus cultures and displayed strong bactericidal activity against persister bacteria. Spontaneous resistance mutation frequency was lower than $10^{-8}$. Exposure to AM-0016 resulted in rapid collapse of the membrane potential. Imaging revealed deformation of the cell envelope. Conclusion: Targeting the cytoplasmic membrane may be an attractive approach to eliminate persister mycobacteria and slow down the emergence of genetic drug resistance.
\end{abstract}

First draft submitted: 4 December 2015; Accepted for publication: 8 March 2016;

Published online: 9 May 2016

With an estimated 9.6 million new cases and 1.5 million deaths in 2014 tuberculosis (TB), caused by Mycobacterium tuberculosis, remains a global infectious disease threat. Half a million multidrugresistant cases compound the situation [1]. There is an urgent medical need for new drugs with new mechanisms of action to keep drug-resistant bacteria at bay. In addition to the emergence of genetic drug resistance, persistence of infection despite extensive chemotherapy represents a second major issue in TB chemotherapy. Shortening treatment time from currently 6-24 months to 2 months or less is the main objective of TB drug development $[2,3]$.

TB drugs target specific macromolecules, either proteins or ribosomal RNA. The major mechanism for mycobacterial antibiotic resistance is mutations in target genes preventing binding of the drugs. Persistence of infection is believed to be due to nongrowing drug-tolerant 'persister' bacteria. The biosynthetic targets of current drugs are required for growth, but less so for survival of metabolically quiescent nonreplicating organisms, thus rendering the bacteria phenotypically drug tolerant [4]. In addition, nongrowing bacilli were shown to have reduced drug uptake [5]. These persister bacilli may pre-exist within growing cell populations, be induced by drug exposure, or they may be generated by suboptimal microenvironmental conditions in vivo [4]. Several culture models are employed to grow drug-tolerant bacilli in vitro. One such model exposes aerobically growing culture to drugs [6]. Many TB agents, such as isoniazid, show an initial rapid kill after which the

'Department of Microbiology \& Immunology, Yong Loo Lin School of Medicine, National University of Singapore, 5 Science Drive 2,

Singapore 117545

${ }^{2}$ Singapore Eye Research Institute, The Academia, 20 College Road, Discovery Tower Level 6, Singapore 169856

${ }^{3}$ SRP Neuroscience \& Behavioural Disorders, Duke-NUS Graduate Medical School, Singapore 169857

${ }^{4}$ Department of Ophthalmology, Yong Loo Lin School of Medicine, National University of Singapore, Singapore 119074

*Author for correspondence: Tel.: +65 6601 1018; Fax: +65 6776 6872; thomas_dick@nuhs.edu.sg

\section{KEYWORDS}

- drug resistance $\bullet$ drug tolerance $\bullet$ tuberculosis 
cidal activity levels off, thus generating a biphasic time kill curve and leaving intact a small subpopulation of nongrowing drug-tolerant persister bacilli [6]. A second widely used mycobacterial persister model exposes the obligate aerobe to hypoxia ('Wayne model') [7], a microenvironmental condition demonstrated for TB lesions in vivo [8]. Upon oxygen starvation, the whole growing culture shifts down to a state of nonreplicating persistence and becomes phenotypically drug resistant. A current working model suggests that we need to kill those two persister types in order to achieve treatment shortening $[4,9]$. The question is what are possible molecular targets required for maintaining viability of nongrowing bacteria with largely reduced metabolism?

The bacterial cytoplasmic membrane is essential irrespective of the metabolic status of the cell. It provides the selective permeability barrier and is the site for a number of critical cellular processes [10]. The large number of antimicrobial peptides made by the host, human defensins for example, validates the membrane as an antibacterial target site. Successful clinical use of a few membrane-targeting antibiotics, such as the lipopetpide daptomycin against Staphylococcus aureus, supports this notion. Furthermore, membranetargeting antibiotics are expected to be slow in resistance development as the membrane is the product of complex biosynthetic pathways, as opposed to a single macromolecule. Again a notion supported by the slow emergence of resistance against membrane-targeting antibiotics used in the clinic [10].

We have recently shown that $\alpha$-mangostin, a natural product from the fruit of Garcinia mangostana, showed potent activity against Grampositive bacteria [11]. In an effort to increase selectivity for the bacterial membrane, we introduced cationic modifications into this compound to yield amphiphilic structures. With AM-0016 (Figure 1), we identified a new Gram-positive antibacterial with good selectivity and low toxicity upon topical as well as systemic application [12]. Biophysical analyses and molecular simulations revealed that AM-0016 targets the bacterial membrane and causes a collapse of the membrane potential. The compound did not show activity against Gram-negative bacteria [12,13].

Here, we first demonstrate potent growth inhibitory and cidal activity of AM-0016 against Mycobacterium tuberculosis and its attenuated, BSL2-compatible, relative Mycobacterium bovis Bacillus Calmette-Guérin (BCG). Then we use this new antibacterial as a tool compound to test the hypothesis that targeting the mycobacterial membrane, as opposed to a specific macromolecular target, may be a useful approach to achieving both killing persister bacilli and delaying the emergence of resistance.

\section{Materials \& methods \\ - Bacterial strains}

Mycobacterium tuberculosis H37Rv (ATCC 27294), M. bovis BCG (ATCC 35734), $M$. smegmatis $\mathrm{mc}^{2} 155$ (ATCC 700084), M. chelonae (ATCC 19539), M. gordonae (ATCC 35756), M. senegalense (ATCC 35755), $M$. intracellulare (ATCC 13950), M. xenopi (ATCC 19250), M. szulgai (ATCC 23069), M. peregrinum (ATCC 23001), $M$. porcinum (ATCC 33775), M. fortuitum (ATCC 6841), M. septicum (ATCC 70071) were used in this study.

\section{- Media \& cultivation}

Liquid cultures were grown in Middlebrook $7 \mathrm{H} 9$ broth (BD Difco) supplemented with $10 \%$ albumin-dextrose-catalase (BD Difco, MD, USA), $0.5 \%$ glycerol (Fisher Scientific, Loughborough, UK) and $0.05 \%$ Tween- 80 (Sigma-Aldrich MI, USA). Solid cultures were grown on Middlebrook $7 \mathrm{H} 10$ agar (BD Difco) supplemented with $0.5 \%$ glycerol and $10 \%$ oleic acid-albumin-dextrose-catalase (OADC) (BD Difco). Liquid cultures were grown in T25 flasks (SPL Life Sciences, Pocheon-si, South Korea) or $14 \mathrm{ml}$ round bottom tubes (SPL Life Sciences) at $37^{\circ} \mathrm{C}$ with agitation as described previously [14]. To generate hypoxic nongrowing cultures, bacteria were grown in Dubos broth (BD Difco) supplemented with Dubos Medium Albumin (BD Difco) in sealed glass tubes with stirring as described previously [15].

\section{- Antibacterials}

AM-0016 was synthesized as previously described [12]. Isoniazid was obtained from Sigma-Aldrich. Isoniazid was dissolved in $90 \%$ DMSO and AM-0016 was dissolved in 20\% DMF. The final concentration of DMSO/DMF in each experiment was below $2 \%$. This concentration of solvent was tested to ensure it had no effect on the growth of all strains tested.

\section{- Minimum inhibitory concentration determination}

Minimum inhibitory concentrations (MICs) were determined in broth or on solid medium 


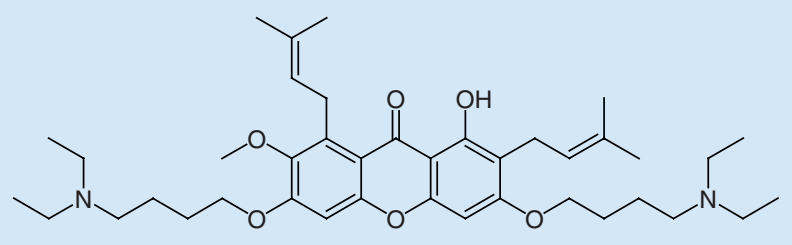

Figure 1. Structure of $\alpha$-mangostin derivative AM-0016.

as described previously [14,16,17]. For both MIC determinations exponentially growing precultures were diluted to $\mathrm{OD}_{600}=0.05$ and drugs were added to $1 \mathrm{ml}$ of cultures in twofold dilutions in $14 \mathrm{ml}$ round bottom tubes. The cultures were incubated for 5 days with shaking at $200 \mathrm{rpm}$ and then visually inspected. $\mathrm{OD}_{600}$ was also measured for each tube. MIC was the lowest concentration that did not show any turbidity [16]. For agar MIC determination $10^{4}$ CFU of exponentially growing precultures were streaked on agar containing twofold dilutions of AM-0016. The cultures were grown for 2-4 days for rapid growers and 1-3 weeks for slow growers and then visually inspected. The 'Agar

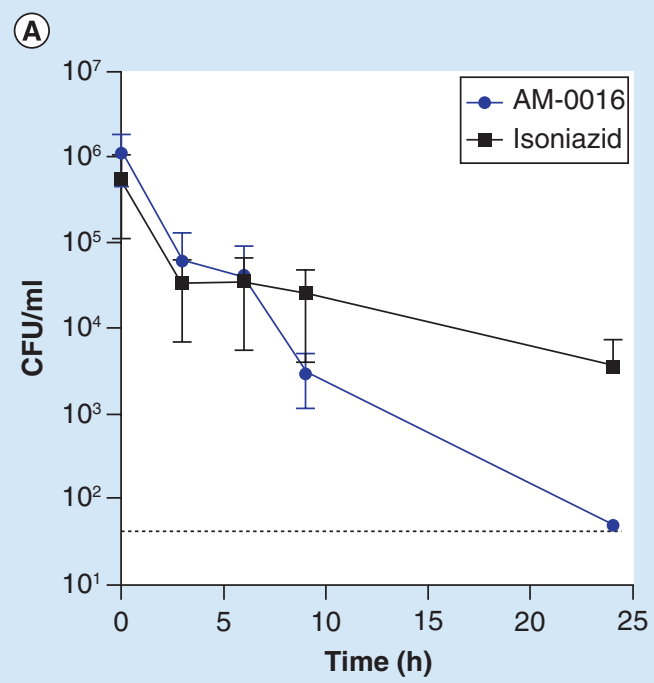

MIC' was the lowest concentration of drug that showed more than $99 \%$ inhibition of growth as compared with the drug-free control [17].

\section{- Determination of cidal activities}

Cidal activities, minimal bactericidal concentration $(\mathrm{MBC})$ for growing bacteria and Wayne cidal concentration (WCC) for hypoxic nongrowing bacilli were determined as described previously [14,16]. Exponentially growing cultures or hypoxic, 20-day-old nongrowing cultures were exposed to various drug concentrations for 5 days and then plated for CFU determination. $\mathrm{MBC}$ and WCC were the lowest concentrations of drug that killed $99.9 \%$ of the initial inoculum.

(B)

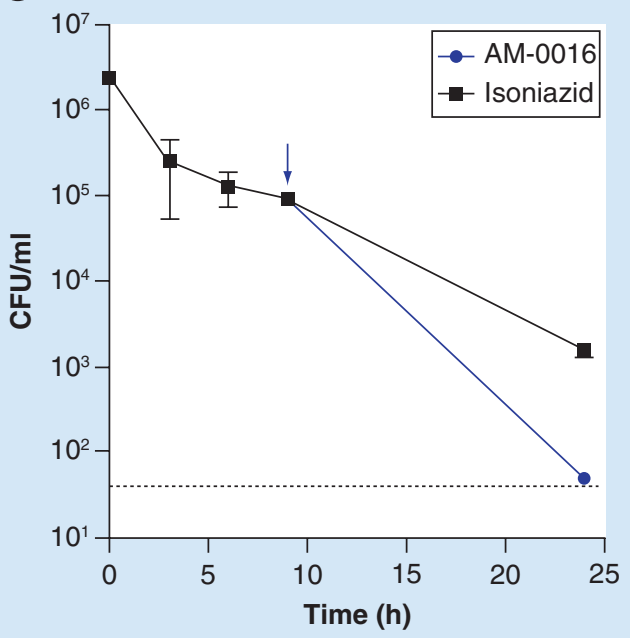

Figure 2. Time kill curves of Mycobacterium bovis Bacillus Calmette-Guérin cultures treated with AM-0016 in comparison with isoniazid. (A) Exponentially growing cultures of Mycobacterium bovis BCG were exposed to $2 \times$ minimum inhibitory concentration of AM-0016 ( $8 \mu \mathrm{M})$ (blue line), or $8 \times$ minimum inhibitory concentration $(50 \mu \mathrm{M})$ of isoniazid (black line) and their effect on viability was determined by CFU monitoring over time. (B) Exponentially growing M. bovis Bacillus CalmetteGuérin were exposed to $50 \mu \mathrm{M}$ isoniazid as before (black line). After $9 \mathrm{~h}$ (arrow), in other words, after the kill curve had leveled off, a sample of the isoniazid containing culture was in addition treated with $8 \mu \mathrm{M} \mathrm{AM-0016}$ (blue line). Limit of detection $(50 \mathrm{CFU} / \mathrm{ml}$ ) is indicated by the horizontal line. Means and standard deviations of three experiments are shown. 


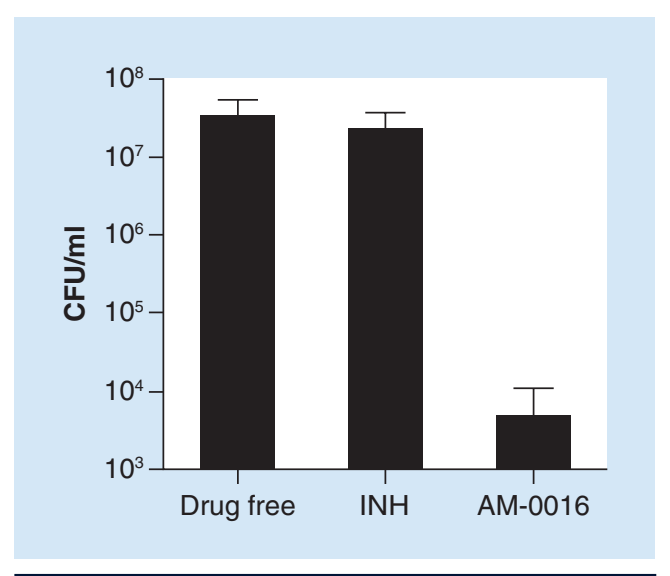

Figure 3. Cidal activity of AM-0016 in comparison with isoniazid against hypoxic nongrowing Mycobacterium bovis Bacillus Calmette-Guérin cultures. 20-day-old hypoxic nongrowing cultures of Mycobacterium bovis Bacillus Calmette-Guérin were exposed to $8 \times$ minimum inhibitory concentration of AM-0016 $(32 \mu \mathrm{M})$ or $16 \times$ minimum inhibitory concentration $(100 \mu \mathrm{M})$ of INH for 5 days and the effect on viability was determined by CFU counting. Means and standard deviations of three experiments are shown.

$\mathrm{INH}$ : Isoniazid.

- Determination of spontaneous resistance mutation frequencies

Mycobacterium bovis BCG or M. smegmatis were grown to mid log phase $\left(\mathrm{OD}_{600}=0.4-0.6\right)$ from which $10^{7} \mathrm{CFU}$ were plated on $7 \mathrm{H} 10$ agar plates containing various concentrations of AM-0016. Plates were incubated for a total of 4 weeks (M. bovis BCG) or 1 week (M. smegmatis) after which apparently resistant colonies were re-streaked on AM-0016 containing agar to confirm resistance. The spontaneous resistance frequency is the ratio of the number of resistance confirmed colonies growing on drug-containing agar to the total number of CFU plated [17].

\section{- Membrane potential assay}

The effect of AM-0016 on the membrane potential of $M$. bovis BCG was measured using the Baclight Bacterial Membrane Potential Kit (Life Technologies, CA, USA) according to the supplier's manual and as described previously [18]. Briefly, $\mathrm{red} / \mathrm{green}$ fluorescence ratios were measured using a Tecan infinite M200 PRO plate reader. Mycobacterium bovis BCG cultures were treated with 1× MIC of AM-0016 and samples were collected at different time points. The cultures were then incubated with $30 \mu \mathrm{M}$ of membrane potential indicator dye Diethyloxacarbocyanine iodide (DiOC2(3)) for $30 \mathrm{~min}$ with $100 \mu \mathrm{M}$ of the proton ionophore Carbonyl cyanide m-chlorophenyl hydrazone (CCCP) treated culture as a positive control. The fluorescence ratios of untreated and CCCP-treated cultures were defined as 100 and $0 \%$ membrane potential, respectively.

\section{- Scanning electron microscopy}

Cultures of $M$. bovis BCG were treated with $1 \times$ MIC of AM-0016 and at different time points $(1,3,6,24 \mathrm{~h})$ samples were collected and fixed with $4 \%$ glutaraldehyde in phosphate-buffered saline (Vivantis Technologies, Selangor Darul Ehsan, Malaysia) at $4{ }^{\circ} \mathrm{C}$ overnight. After washing, prefixed samples were postfixed in $1 \% \mathrm{OsO}_{4}$ reagent for $1 \mathrm{~h}$, followed by dehydration in gradually increased concentration of ethanol up to $100 \%$. Next, dehydrated samples were transferred to a critical point dryer for infiltration

$\begin{aligned} & \text { Table 1. Agar minimum inhibitory concentration of AM-0016 against nontuberculous } \\
& \text { mycobacteria. }\end{aligned}$
\begin{tabular}{ll} 
Mycobacterial strain & Agar MIC $(\boldsymbol{\mu M})$ \\
M. chelonae & 2.5 \\
M. gordonae & 5 \\
M. smegmatis & 10 \\
M. senegalense & 10 \\
M. intracellulare & 10 \\
M. xenopi & 30 \\
M. szulgai & 30 \\
M. peregrinum & 30 \\
M. porcinum & 30 \\
M. septicum & 30 \\
M. fortuitum & 30 \\
\hline Agar minimum inhibitory concentration of Mycobacterium tuberculosis and BCG were $10 \mu \mathrm{M}$. \\
\hline
\end{tabular}


with $\mathrm{CO}_{2}$. After mounting the specimen on scanning electron microscopy stubs, samples were sputter-coated with a thin layer of gold and examined using a JEOL JSM-6701F Field Emission Scanning Electron Microscope.

\section{Results}

\section{- AM-0016 has potent growth inhibitory \& cidal activity against $M$. tuberculosis \& M. bovis BCG}

The MIC of AM-0016 against M. tuberculosis $\mathrm{H} 37 \mathrm{Rv}$ was determined by the broth dilution method and was found to be $4 \mu \mathrm{M}$. The same culture assay conditions (i.e., 5 days of exposure, see the 'Materials \& methods' section) were used to determine $\mathrm{MBC}$ of the compound (lowest concentration that causes 1000 -fold kill) and $\mathrm{MBC}$ was found to be equal to the MIC. MIC and MBC were also determined against the attenuated tubercle bacillus $M$. bovis BCG and were found to be identical, in other words, $4 \mu \mathrm{M}$. These results show that AM-0016 has attractive growth inhibition and cidal potency against the tubercle bacillus. In the following $M$. bovis BCG, a BSL2-compatible close relative of $M$. tuberculosis, was employed for the characterization of AM-0016 potencies, resistance development and mechanism of action studies. MICs were also determined in broth with acid $\mathrm{pH}(\mathrm{pH}$ 5.8) and the values were found to be identical to those at standard broth $\mathrm{pH}(\mathrm{pH}$ 6.8).

\section{- AM-0016 kills growing M. bovis BCG cultures fast \& completely}

Isoniazid, targeting mycolic acid synthesis [19] is one of the fast killing TB drugs and shows a typical bi-phasic time kill curve. After an initial phase of rapid kill, the curve levels off and a drugtolerant persister population survives exposure to the drug (Figure 2A). To test the hypothesis that a membrane acting antimycobacterial should kill not only fast, but in contrast to many anti-TB agents, completely, we exposed growing cultures of the tubercle bacillus to $2 \times$ MIC of AM-0016 and determined viability over time by CFU enumeration. Figure 2A shows that AM-0016 killed as fast as isoniazid in the initial phase. In contrast to isoniazid, however, AM-0016's cidal activity did not level off over time and apparently sterilized the culture. These results suggest that AM-0016 kills a growing culture of the tubercle bacillus fast and completely. To determine whether AM-0016 can actually also kill isoniazid-induced persister bacilli, cultures were treated with isoniazid until the kill curve leveled off, leaving intact a drug-tolerant subpopulation of bacilli. Then AM-0016 was added as before. Figure 2B shows that this resulted in the kill of the isoniazid-induced subpopulation of persister organisms.

\section{- AM-0016 kills hypoxic nongrowing M. bovis BCG cultures efficiently}

Hypoxic nongrowing tubercle bacilli show a high level of drug tolerance, in other words, the nonreplicating bacilli are not killed by drugs such as isoniazid, which are highly cidal for growing bacteria. To determine if AM-0016 kills nonreplicating bacilli, we exposed hypoxic nongrowing cultures of $M$. bovis BCG to the compound and measured CFU. Figure 3 shows that isoniazid at $16 \times$ MIC $(100 \mu \mathrm{M})$ had no effect on the number of CFU after 5 days of incubation (the same exposure time used to determine $\mathrm{MBC}$ for growing cultures). In contrast, AM-0016 at

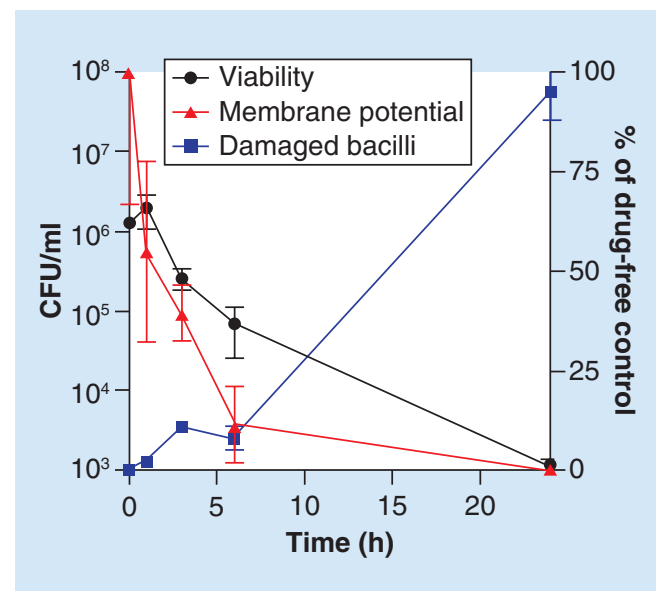

Figure 4. Effect of AM-0016 on the membrane potential and cell envelope of Mycobacterium bovis Bacillus Calmette-Guérin. Growing M. bovis Bacillus Calmette-Guérin culture was exposed to AM-0016 (1× minimum inhibitory concentration) and samples were taken over time to determine the effect on the membrane potential (red triangles; percentage compared with drug-free control cultures). Exposure to Isoniazid at $8 \times$ minimum inhibitory concentration did not affect the membrane potential (data not shown). In parallel, samples were subjected to scanning electron microscopic analysis and the percentage $(n=50)$ of damaged cells (see Figure 5 ) were enumerated (blue squares). Viability over time was determined by CFU measurement (black circles). Means and standard deviations of three experiments are shown. 


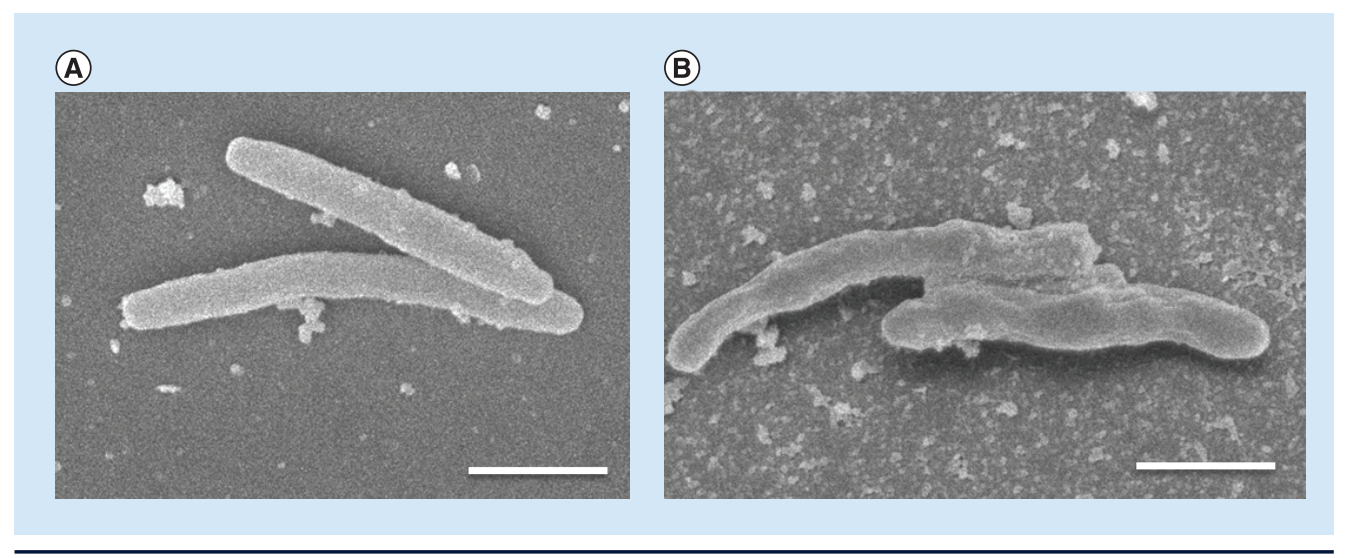

Figure 5. Scanning electron micrographs of Mycobacterium bovis Bacillus Calmette-Guérin after exposure to AM-0016. As described in Figure 4, growing M. bovis BCG culture was exposed to AM-0016 and subjected to electron microscopic analysis. (A) Drug-free control. (B) After 1 day of exposure to $1 \times$ MIC of AM- $001695 \%$ of the bacilli showed the type of depicted cell envelope damage. The scale bar corresponds to $1 \mu \mathrm{m}$.

$8 \times$ MIC showed more than a 1000 -fold reduction in CFU, in other words, the 'Wayne cidal concentration' (WCC), the lowest concentration that reduces the CFU of a hypoxic nongrowing culture by 3 logs, was $32 \mu \mathrm{M}$. These results show that AM-0016 retained an attractive cidal activity against nonreplicating bacteria.

\section{- Mycobacterium bovis BCG shows a low spontaneous resistance mutation frequency against AM-0016}

To determine the spontaneous resistance mutation frequency of mycobacteria against AM-0016 we first carried out a pilot study with the fast growing $M$. smegmatis (agar $\mathrm{MIC}=10 \mu \mathrm{M})$. Plating of $10^{8} \mathrm{CFU}$ on agar plates containing two-, fouror eight-times the MIC of AM-0016 did not yield any resistant colonies, suggesting that the spontaneous resistance mutation frequency was lower than $10^{-8} / \mathrm{CFU}$. Plating of $10^{9} \mathrm{CFU}$ on agar containing $2 \times$ MIC yielded one colony that grew upon re-streaking on agar containing the same AM-0016 concentration. Determination of the actual broth MIC of the AM-0016 resistant $M$. smegmatis strain showed that the MIC had merely doubled compared with the value for wild-type bacilli. Then we grew two independent $M$. bovis BCG cultures and plated $10^{8} \mathrm{CFU}$ each on agar containing AM-0016 at $10 \mu \mathrm{M}$ and obtained one colony that grew upon re-streaking the bacilli on drug containing agar. Broth MIC determination of the AM-0016 resistant $M$. bovis BCG strain showed again an only twofold increase compared to wild-type bacteria. Taken together, these results show that the spontaneous resistance mutation frequency for AM-0016 was lower than 10-8/CFU. Isoniazid resistance, determined for comparison in a parallel experiment, occurred at a frequency of $10^{-6} / \mathrm{CFU}$ in $M$. bovis BCG. Importantly, the level of resistance against AM-0016, a twofold increase in the MIC, was very low. These results suggest that AM-0016 has a low propensity for the development of significant resistance.

\section{- AM-0016 exposure causes rapid collapse of the membrane potential in M. bovis BCG}

We showed previously that AM-0016 kills Staphylococcus aureus via disruption of its membrane potential [12]. To interrogate whether the cidal effect of AM-0016 on mycobacteria is also due to interference with membrane integrity, we determined if AM-0016 acted via reducing the membrane potential using the fluorescent membrane potential indicator dye Diethyloxacarbocyanine iodide, with parallel CFU determination. Figure 4 shows that exposure to AM-0016 caused a rapid collapse of the membrane potential, paralleling loss of viability of the culture. These results suggest that AM-0016 kills the bacillus by disrupting the membrane integrity.

\section{- AM-0016 exposure causes cell envelope damage in $M$. bovis BCG}

To interrogate whether exposure to AM-0016 causes structural damage to the mycobacterial cell envelope, scanning electron microscopic analyses were carried out. Cultures were exposed to AM-0016 and sampled over time. Figure 5 shows that exposure of the bacteria to 
AM-0016 results in distortion of the cell envelope. The quantification of damaged cells over time depicted in Figure 4 shows that the increase in number of compromised cells correlated with a decrease in CFU. These results suggest that AM-0016 interferes with the bacterial cell wall. Whether this damage is due to a direct interaction of AM-0016 with cell wall components or represents a secondary effect due to druginduced collapse of membrane potential remains to be determined. As controls we imaged $M$. bovis BCG cultures treated in a similar way with ciprofloxacin or the un-coupler carbonyl cyanide m-chlorophenyl hydrazine. No significant distortion of cell envelopes was observed under these conditions.

\section{- AM-0016 is active against nontuberculous mycobacteria}

To determine whether AM-0016's antimycobacterial activity is specific to the tubercle bacillus or includes nontuberculous mycobacteria, we carried out agar MIC measurements for a panel of mycobacterial species. Table 1 shows that AM-0016 was active against all species tested, displaying a spectrum of potencies from 2.5 to $30 \mu \mathrm{M}$.

\section{Discussion}

$\mathrm{TB}$ control is plagued by two issues: persistence of infection despite extensive chemotherapy and rapid emergence of drug resistance. Current TB chemotherapy takes so long to achieve cure as it is ineffective in eradicating persister bacilli. The long treatment duration fosters noncompliance of patients, which promotes development of genetically drugresistant strains. New drugs for TB should ideally shorten treatment time and have a low propensity for the development of resistance [4].

Here we tested the hypothesis that antimicrobials targeting the mycobacterial cytoplasmic membrane might fulfill both requirements, in other words, to effectively kill persister bacteria and to show a slow development of resistance. After showing that the anti-Grampositive, membrane-targeting $\alpha$-mangostin derivative AM-0016 possessed growth inhibitory and cidal activity against $M$. tuberculosis, we demonstrated that this new antimycobacterial killed growing tubercle bacillus cultures rapidly and completely, in other words, it did not leave any persister subpopulations intact, showed strong cidal activity against phenotypic drug-tolerant hypoxic nongrowing cultures and had a low spontaneous resistance mutation frequency, with the arising mutants showing a very low level of a resistance. By demonstrating that cidal activity of AM-0016 correlated with collapse of the membrane potential we provide evidence that the mechanism of action of the compound is indeed via impairing membrane integrity of the bacillus. Furthermore, scanning electron microscopy imaging showed that the compound affected the structure of the cell envelope. Finally, we demonstrate that AM-0016 has a wide anti-mycobacterial spectrum.

\section{Conclusion}

Taken together, our work suggests that targeting the mycobacterial cytoplasmic membrane may be an attractive novel approach to eliminate persister mycobacteria and to slow down the development of genetic drug resistance.

\section{Future perspective}

Our in vitro proof-of-concept studies with the tool compound AM-0016 suggest that the membrane as target may represent an attractive novel chemotherapeutic intervention level for the discovery of treatment shortening antimycobacterials. Lead optimization efforts are in progress to introduce the required pharmacokinetic properties, including oral bioavailability [14], into this xanthone scaffold for the development compounds suitable for efficacy studies in mice.

\section{Acknowledgements}

We would like to thank Martin Gengenbacher, National University of Singapore, for discussion and comments on the manuscript and Josephine Howe, National University of Singapore, for her help with electron microscopy. We thank the Yong Loo Lin School of Medicine BSL3 Core Facility for support.

Financial \& competing interests disclosure

This work was supported by the Singapore Ministry of Health's National Medical Research Council under its Translational Clinical Research Flagship grant (NMRC/TCR/011NUHS/2014) and its Centre grant 'MINE'/Research Core number 4 (NMRC/CG/013/2013) to T Dick, SHF/FG538P/2013-SingHealth Foundation to S Liu, NMRC/TCR/R1018 to R Beuerman and is part of the Singapore Programme of Research Investigating New Approaches to Treatment of Tuberculosis (SPRINT-TB; www.sprinttb.org) led by Nick Paton. D Mukherjee received a research scholarship from Yong Loo Lin School of Medicine. The authors have no other relevant affiliations or financial 
interests in or financial conflicts with the subject matter or materials discussed in the manuscript apart from those disclosed.

No writing assistance was utilized in the production of this manuscript.

\section{Open access}

This work is licensed under the AttributionNonCommercial-NoDerivatives 4.0 Unported License. To view a copy of this license, visit http://creativecommons.org/ licenses/by-nc-nd/4.0/

\section{EXECUTIVE SUMMARY}

- Current anti-mycobacterials have distinct proteins or ribosomal RNAs as their target.

- Persistence of infection due to drug-tolerant persister bacilli and development of genetic drug resistance represent major issues in current therapies of mycobacterial infections.

- Using the xanthone-based AM-0016 as a tool compound we show that targeting the mycobacterial membrane results in efficient killing of persister bacilli. Furthermore, no significant drug resistance development could be observed.

- These results suggest that drugs targeting the membrane may be useful for the development of treatment shortening anti-mycobacterial regimens and for delaying the emergence of genetic resistance.

\section{References}

1 World Health Organisation. Global Tuberculosis Report 1-192 (2015).

2 Barry CE, Boshoff HI, Dartois V et al. The spectrum of latent tuberculosis: rethinking the biology and intervention strategies. Nat. Rev. Microbiol. 7(12), 845-855 (2009).

3 Gengenbacher M, Kaufmann SH. Mycobacterium tuberculosis: success through dormancy. FEMS Microbiol. Rev. 36(3), 514-532 (2012).

4 Dartois V, Leong FJ, Dick T. Tuberculosis drug discovery: issues, gaps and the way forward. In: Antiparasitic and Antibacterial Drug Discovery: From Molecular Targets to Drug Candidates (Chapter 21). Selzer PM (Ed.). Wiley-VCH Verlag GmbH \& Co. KGaA, Weinheim, Germany, 415-440 (2009).

5 Sarathy J, Dartois V, Dick T, Gengenbacher M. Reduced drug uptake in phenotypically resistant nutrient-starved nonreplicating Mycobacterium tuberculosis. Antimicrob. Agents Chemother. 57(4), 1648-1653 (2013).

6 Keren I, Minami S, Rubin E, Lewis K. Characterization and transcriptome analysis of Mycobacterium tuberculosis persisters. MBio 2(3), (2011).

7 Wayne LG, Hayes LG. An in vitro model for sequential study of shiftdown of
Mycobacterium tuberculosis through two stages of nonreplicating persistence. Infect. Immun. 64(6), 2062-2069 (1996).

8 Lenaerts A, Barry CE 3rd, Dartois V. Heterogeneity in tuberculosis pathology, microenvironments and therapeutic responses. Immunol. Rev. 264(1), 288-307 (2015).

9 Dick T. Dormant tubercle bacilli: the key to more effective TB chemotherapy? J. Antimicrob. Chemother. 47(1), 117-118 (2001).

10 Hurdle JG, O’Neill AJ, Chopra I, Lee RE. Targeting bacterial membrane function: an underexploited mechanism for treating persistent infections. Nat. Rev. Microbiol. 9(1), 62-75 (2011).

11 Koh JJ, Qiu S, Zou H et al. Rapid bactericidal action of alpha-mangostin against MRSA as an outcome of membrane targeting. Biochim. Biophys. Acta 1828(2), 834-844 (2013).

12 Zou H, Koh JJ, Li J et al. Design and synthesis of amphiphilic xanthone-based, membrane-targeting antimicrobials with improved membrane selectivity. J. Med. Chem. 56(9), 2359-2373 (2013).

13 Koh JJ, Lin S, Aung TT et al. Amino acid modified xanthone derivatives: novel, highly promising membrane-active antimicrobials for multidrug-resistant Gram-positive bacterial infections. J. Med. Chem. 58(2), 739-752 (2015).
14 Lakshminarayana SB, Huat TB, Ho PC et al. Comprehensive physicochemical, pharmacokinetic and activity profiling of antiTB agents. J. Antimicrob. Chemother. 70 (3), 857-867 (2015).

15 Lim A, Eleuterio M, Hutter B, Murugasu-Oei B, Dick T. Oxygen depletion-induced dormancy in Mycobacterium bovis BCG. J. Bacteriol. 181(7), 2252-2256 (1999).

16 Murugasu-Oei B, Dick T. Bactericidal activity of nitrofurans against growing and dormant Mycobacterium bovis BCG. J. Antimicrob. Chemother. 46(6), 917-919 (2000).

17 Gopal P, Dick T. The new tuberculosis drug Perchlozone ${ }^{\circledR}$ shows cross-resistance with thiacetazone. Int. J. Antimicrob. Agents 45(4), 430-433 (2015).

18 Rao SPS, Alonso S, Rand L, Dick T, Pethe K. The protonmotive force is required for maintaining ATP homeostasis and viability of hypoxic, nonreplicating Mycobacterium tuberculosis. Proc. Natl Acad. Sci. USA 105(33), 11945-11950 (2008).

19 Banerjee A, Dubnau E, Quemard A et al. inhA, a gene encoding a target for isoniazid and ethionamide in Mycobacterium tuberculosis. Science 263(5144), 227-230 (1994). 